\title{
The three R's of lung health and disease: repair, remodeling, and regeneration
}

\author{
Michael F. Beers ${ }^{1,2}$ and Edward E. Morrisey $1,2,3,4$
}

1Department of Medicine, ${ }^{2}$ Institute for Regenerative Medicine, ${ }^{3}$ Cardiovascular Institute, and ${ }^{4}$ Department of Cell and Developmental Biology, University of Pennsylvania School of Medicine, Philadelphia, Pennsylvania, USA.

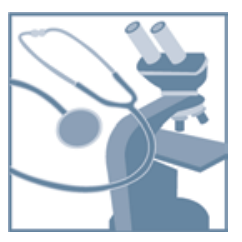

All tissues and organs can be classified according to their ability to repair and regenerate during adult homeostasis and after injury. Some exhibit a high rate of constant cell turnover, while others, such as the lung, exhibit only low-level cell regeneration during normal adult homeostasis but have the ability to rapidly regenerate new cells after injury. Lung regeneration likely involves both activation of progenitor cells as well as cell replacement through proliferation of remaining undamaged cells. The pathways and factors that control this process and its role in disease are only now being explored. In this Review, we will discuss the connection between pathways required for lung development and how the lung responds to injury and disease, with a particular emphasis on recent studies describing the role for the epithelium in repair and regeneration.

\section{Introduction}

Repair, remodeling, and regeneration of the respiratory system for the restoration of normal function after injury represent the holy grail of modern pulmonary biology. As with other externally exposed organs, such as the skin, lung function requires the activity of both the innate and acquired immune systems to monitor exogenous insults and injury. However, the lung has the capacity to perform significant amounts of xenobiotic metabolism, making it susceptible to a variety of insults and injuries, both exogenous and endogenous. The lung is extremely complex, and both development and repair require interaction among more than 40 different cell lineages (for review see refs. 1-3). The complex nature of both lung structure and injury repair mechanisms impact the ability of the lung to affect efficient repair.

The complex spatial organization of the lung and the sheer variety of cells that constitute the mature organ dictate that repair after injury may require multiple pools of progenitor cells capable of self renewal and multipotency. These progenitor cells are thought to reside in specialized niches within the lung (3-7). Much of what we know about them has come from the study of the basic developmental biology of the lung in model organisms such as the mouse. Therefore, an improved understanding of both developmental biology and progenitor/stem cell biology, as it pertains to the mature lung and the generation of various cell types within the alveolar and bronchiolar airways, is paramount to the development of better therapies for both acute and chronic lung disease.

This Review will examine current concepts related to the cellular strategies for repair, remodeling, and regeneration of the injured lung. We will focus particular attention on the epithelial cellular component, as it is both a primary target of external injury and a key lineage in the repair process critical for reestablishment of normal pulmonary function. We also make the distinction between regeneration, which occurs with the successful restoration of a functional epithelial-lined airway and alveolar airspaces, and simple repair, which can include scar formation, injuryinduced fibrosis, and aberrant repopulation of segments of the

Conflict of interest: The authors have declared that no conflict of interest exists. Citation for this article: J Clin Invest. 2011;121(6):2065-2073. doi:10.1172/JCI45961. respiratory tract with dysfunctional epithelium. Our attempt is not to provide an exhaustive overview of lung development, adult lung injury, or the pathways involved, as these have been covered in several earlier reviews $(1,3,8,9)$, but rather to point out the similarities between what occurs during the developmental process and in the injury response in the lung required to properly regenerate damaged and lost cell lineages. A unifying theme that becomes clear from this overview is that damage to the adult lung and ensuing cell drop-out, whether it be from acute lung injury (ALI) or chronic diseases, results in substantial alterations in lung epithelial cell homeostasis. The connection between basic pathways and the reparative responses by the injured or damaged lung as well as recent seminal observations describing the ability of the lung to use endogenous progenitor cell populations to regenerate epithelia will be explored.

\section{Lung development}

The mammalian lung develops rather late in gestation, with the earliest signs of the budding endoderm observed at about four weeks in humans and at about E9.5 in the mouse (Figure 1A and ref. 3). This endoderm is surrounded by mesenchymal cells derived from the splanchnic mesoderm, which will later contribute to the development of the pulmonary vascular system (endothelial and vascular smooth muscle cells), airway smooth muscle, and pulmonary fibroblasts. The early lung endoderm is rather plastic and can adopt either distal cell fates or proximal cell fates depending on paracrine signaling from the surrounding mesenchyme (10, 11). However, lung endoderm plasticity is temporally restricted to development before E13.5 in the mouse, as has been recently shown by temporally restricted fate-mapping experiments (6). Thus, a complex interplay between endoderm and mesoderm cell types helps to define early developmental competence and cell fate in the lung. These interactions are also critical for lung repair and regeneration as will be discussed below.

The proximal-distal patterning of the lung is accompanied by the gradual restriction of the ability of developmental progenitors to generate the various epithelial lineages in the mature organ (Figure 1). During the earliest stages of lung development, SRY-box containing gene 9 (Sox9) and inhibitor of DNA binding 2 (Id2) mark the 
A

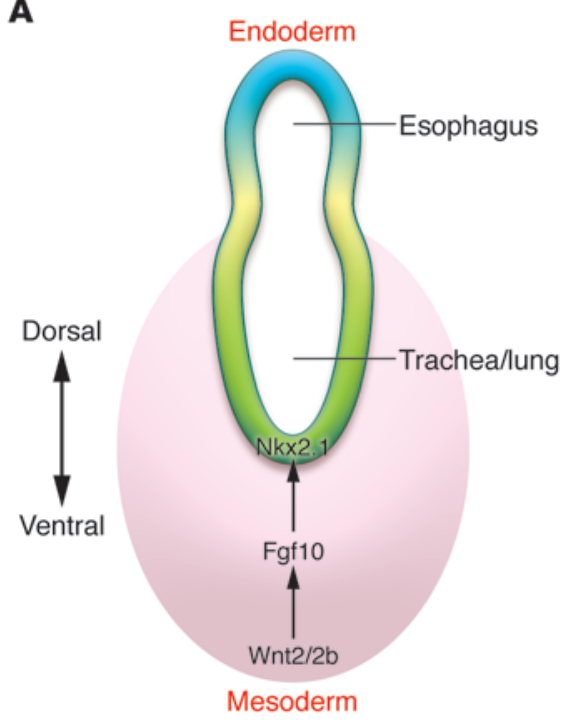

B

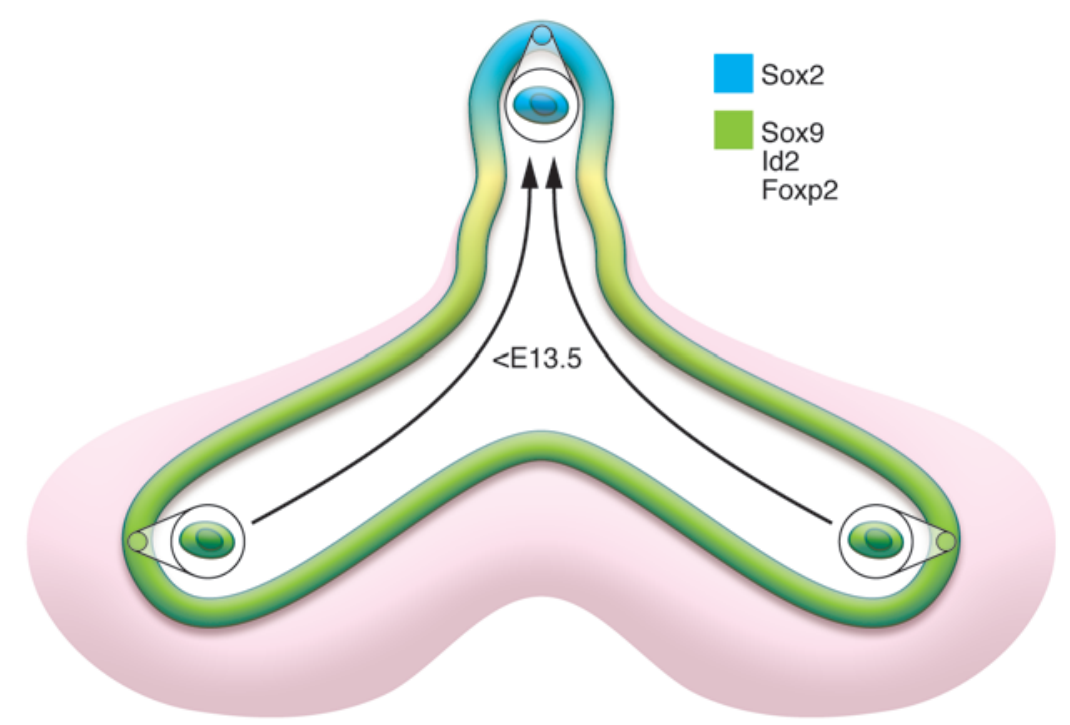

\section{Figure 1}

Early patterning and morphogenesis of the anterior foregut and origin of epithelial cell lineages in the lung. (A) Diagram of the early anterior foregut development, showing the relationship between Wnt2/2b and Fgf10, which are expressed in the early ventral anterior mesoderm surrounding the foregut. Wnt2/2b act upstream of Fgf10 (and other factors including Bmp4) to induce lung specification in the most ventral aspect of the anterior foregut endoderm, as noted by expression of Nkx2.1. (B) As lung development ensues, the endoderm goes through extensive and reiterated branching, setting up the proximal-distal patterning of the airways. This results in distinct progenitors located within the proximal $\left(\right.$ Sox2 $\left.{ }^{+}\right)$and distal (Sox9, Id2, Foxp2) airway epithelium. However, the distal Id2 ${ }^{+}$progenitors can generate proximal epithelium up to E13.5 of mouse development (B, arrows) (6).

distal endoderm progenitor population, while Sox 2 marks the proximal endoderm progenitor population (12-15). Id $2^{+}$distal progenitors have the ability to contribute to the proximal epithelium up to E13.5, as shown by lineage fate-mapping analysis (6). After this time point, $\mathrm{Id} 2^{+}$distal progenitors become restricted in their fate and only contribute to distal epithelium and ultimately alveolar epithelium. These findings suggest that early lung endoderm progenitors are multipotent and may represent a useful cell lineage for comparison purposes in establishing the multipotent nature of adult progenitors and their regenerative capacity in the adult lung. A summary of different progenitor lineages and genes that mark them is shown in Table 1.

As mentioned above, epithelial-mesenchymal interactions help to pattern the lung epithelium in a distinct proximal-distal manner, which is required for the proper differentiation of epithelial cell types essential for postnatal respiration. Several signaling pathways regulate epithelial-mesenchymal interactions in the lung, including Wnt, Bmp, TGF- $\beta$, and Fgf. Of these, the Wnt signaling pathway is of interest due to its role in both lung development and in regulation of progenitor cells within the lung. Wnts are a large family of 19 secreted cysteine rich ligands that bind to a coreceptor complex composed of members of the Frizzled family and the LRP5/ 6 transmembrane proteins. Wnts can signal through multiple pathways, and the best understood of these is the canonical or $\beta$-catenin dependent pathway. In this pathway, Wnt binding to Frizzled/LRP coreceptors leads to inhibition of the kinase GSK3 $\beta$. $\beta$-Catenin is a target of GSK3 $\beta$, and when phosphorylated at key residues in its amino terminal end, $\beta$-catenin protein undergoes proteosome-mediated degradation. Thus, active Wnt signaling causes $\beta$-catenin to be hypophosphorylated, resulting in its stabi- lization, accumulation, and translocation to the nucleus, at which point it binds members of the LEF/TCF transcription factor family to activate target gene expression (for review see ref. 16).

Canonical Wnt signaling is active at high levels in early lung development primarily in the primitive endoderm $(17,18)$. As lung development progresses, Wnt signaling activity decreases in the epithelium, but activity is initiated in the developing airway smooth muscle precursors $(17,19)$. Mouse genetic models have implicated this pathway in several key roles in the developing lung. Loss of $W n t 2 / 2 b$ leads to a complete loss of lung endoderm progenitor specification and failure of lung and trachea formation (20). This phenotype can be mimicked by deletion of $\beta$-catenin in the anterior foregut endoderm prior to lung specification in the mouse $(20,21)$. Conversely, activation of Wnt/ $\beta$-catenin signaling in the anterior foregut endoderm leads to an expansion of the lung endoderm progenitor cell fate into the presumptive esophagus and stomach regions $(20,21)$. Loss of $\beta$-catenin after lung specification leads to a loss in distal airway epithelial differentiation (22). This is concordant with a loss in Fgf and Bmp signaling, suggesting that Wnt signaling acts fairly high in a hierarchy of pathways required for early lung development (17). In agreement with these observations, Fgf10, Fgfr2, and Bmp4 have been shown to be direct transcriptional targets of $\mathrm{Wnt} / \beta$-catenin signaling $(17,23)$. These studies support the concept that the early foregut endoderm is rather plastic in its fate and that Wnt/ $\beta$-catenin signaling can dominantly impose a lung endoderm progenitor fate during a specific temporal window of development (Figure 1). Such findings may have important implications in programming cell lineages to a lung endoderm fate, such as in generating lung epithelium from pluripotent stem cells. 


\section{Table 1}

Cell lineage markers of potential respiratory progenitors

\section{Distal epithelial progenitors}

$\begin{array}{lc}\text { Gene } & \text { Protein classification } \\ \text { Sox9 } & \text { High-mobility group transcription factor } \\ \text { Id2 } & \text { bHLH transcription factor } \\ \text { Foxp2 } & \text { Forkhead transcription factor } \\ \text { Proximal epithelial progenitors } \\ \text { Sox2 } & \text { High-mobility group transcription factor } \\ \text { Tp63 } & \text { bHLH-leucine zipper transcription factor } \\ \text { Krt5 } & \text { Epidermal keratin } \\ \text { Krt14 } & \text { Epidermal keratin }\end{array}$

\section{Embryonic expression}

Yes, tips of branching airways Yes, tips of branching airways Yes, tips of branching airways

Yes, proximal epithelium
of the branching airways
Yes, basal cells in proximal bronchi
and trachea
Yes, similar to Tp63
Yes, similar to Tp63

$\begin{array}{cc}\text { Adult expression } & \text { Refs. } \\ \text { Some low level expression } & 13 \\ \text { No } & 6 \\ \text { Yes, AEC1 and AEC2 cells } & 32\end{array}$

Yes, proximal secretory epithelium of bronchiolar and tracheal airways Yes, basal cells in proximal bronchi and trachea

Yes, similar to Tp63

Yes, similar to Tp63
$14,15,70$

\section{5,7 \\ 5,89}

bHLH, basic helix-loop-helix.

Other pathways, including Bmp and Fgf signaling, are important players in the development of the lung, and, as mentioned above, they are often transcriptional targets of Wnt/ $\beta$-catenin signaling. Fgf signaling through multiple ligands, including Fgf7, Fgf9, and Fgf10, regulates the proliferation and differentiation of both epithelial and mesenchymal compartments in the lung. Loss of Fgf10 leads to a severe truncation of lung airway branching, although specification is not affected $(24,25)$. This phenotype is similar to a loss of Fgfr2 expression in the developing embryo, suggesting that this is the primary receptor for Fgf10 in the lung (26). Fgf10 is also a direct target of Wnt/ $\beta$-catenin signaling, providing an important signaling axis regulating early lung endoderm specification and growth (23). Fgf9 affects both epithelial and peripheral mesenchymal proliferation $(27,28)$. In the case of Bmp signaling, several reports have demonstrated that a block in Bmp signaling, through expression of secreted inhibitors such as noggin or gremlin or dominant-negative receptors, leads to a loss in proximal-distal patterning of the $\operatorname{lung}(29,30)$. Genetic deletion of the receptor Bmprla in the developing lung epithelium leads to decreased epithelial proliferation and increased epithelial cell death, indicating a role for Bmp signaling in growth and maintenance of the developing lung epithelia (31).

Lung development continues postnatally in mammals with the maturation of the alveolar airspaces. This alveolarization process includes differentiation of both alveolar epithelial type 1 (AEC1) cells and AEC2 cells to increase gas exchange capacity and generate surfactant required to reduce surface tension within the alveolar airspace. As with embryonic lung development, multiple transcription factors have been implicated in regulating alveolarization, including Gata6, Nkx2.1, and Foxp2 (32-34). Generally, expression of these factors decreases in the postnatal lung, and gain-of-function studies in transgenic mice have demonstrated that persistent overexpression of Gata 6 and Nkx2.1 leads to defective alveolarization $(33,34)$. These studies show that the embryonic program is not only critical for lung development but that precisely regulated expression of these factors is necessary so that high levels are achieved during early development to promote rapid expansion and differentiation of embryonic progenitors and suppression during late gestation and postnatal development to allow for proper maturation of the lung epithelium.

\section{Injury responses in the adult lung}

Although many critical factors are known to regulate development of the lung, less is understood about the factors and pathways required for proper epithelial repair and regeneration following injury. This is particularly important given the evidence that developmental pathways are often recapitulated in adult injury repair induced during disease states (35). In humans, the program regulating pulmonary cell homeostasis is altered in most pulmonary disease states following injury to the mature lung (Figure 2). After injury, lung epithelium may either activate the necessary repair and regeneration pathways for proper repopulation of lost epithelial cells or undergo an aberrant remodeling and differentiation process, which represents the final common pathway for many types of parenchymal pulmonary diseases (Figure 2). Diseases marked by this dysfunctional repair and remodeling span the spectrum from chronic interstitial lung diseases, including idiopathic interstitial pneumonia, autoimmune-related fibrosis, and bleomycin pulmonary toxicity, to the chronic obstructive pulmonary diseases (COPDs), including emphysema and chronic asthma as well as late phases of ALI/adult respiratory distress syndrome (ALI/ARDS) and cystic lung remodeling, such as Birt-Hogg-Dube syndrome (36). Many of these diseases involve defective epithelialmesenchymal interactions, leading to dysfunctional wound healing and resulting in abnormal lung remodeling $(35,37,38)$. The concept that abnormal repair can lead to defective tissue remodeling can be extended to pulmonary vascular diseases, which likely represent a similar parallel of endothelial-mesenchymal dysfunction. This has been extensively reviewed elsewhere (39).

The events in the pathogenesis of parenchymal lung disease and injury can be divided into three basic phases, which are shown in Figure 3. These phases are as follows: initiation, resulting from intrinsic and/or extrinsic events to produce epithelial dysfunction and damage; amplification, mediated in part by the resulting inflammatory response; and the response stage, in which epithelial cells undergo a decision to either repair appropriately via activation of proliferative and differentiation programs or undergo inappropriate remodeling, which might include excessive apoptosis, epithelial-mesenchymal transition, or reprogramming to a defective and dysfunctional state of differentiation. This is the critical branch point at which either successful correction of damaged epithelium ensues or abnormal 


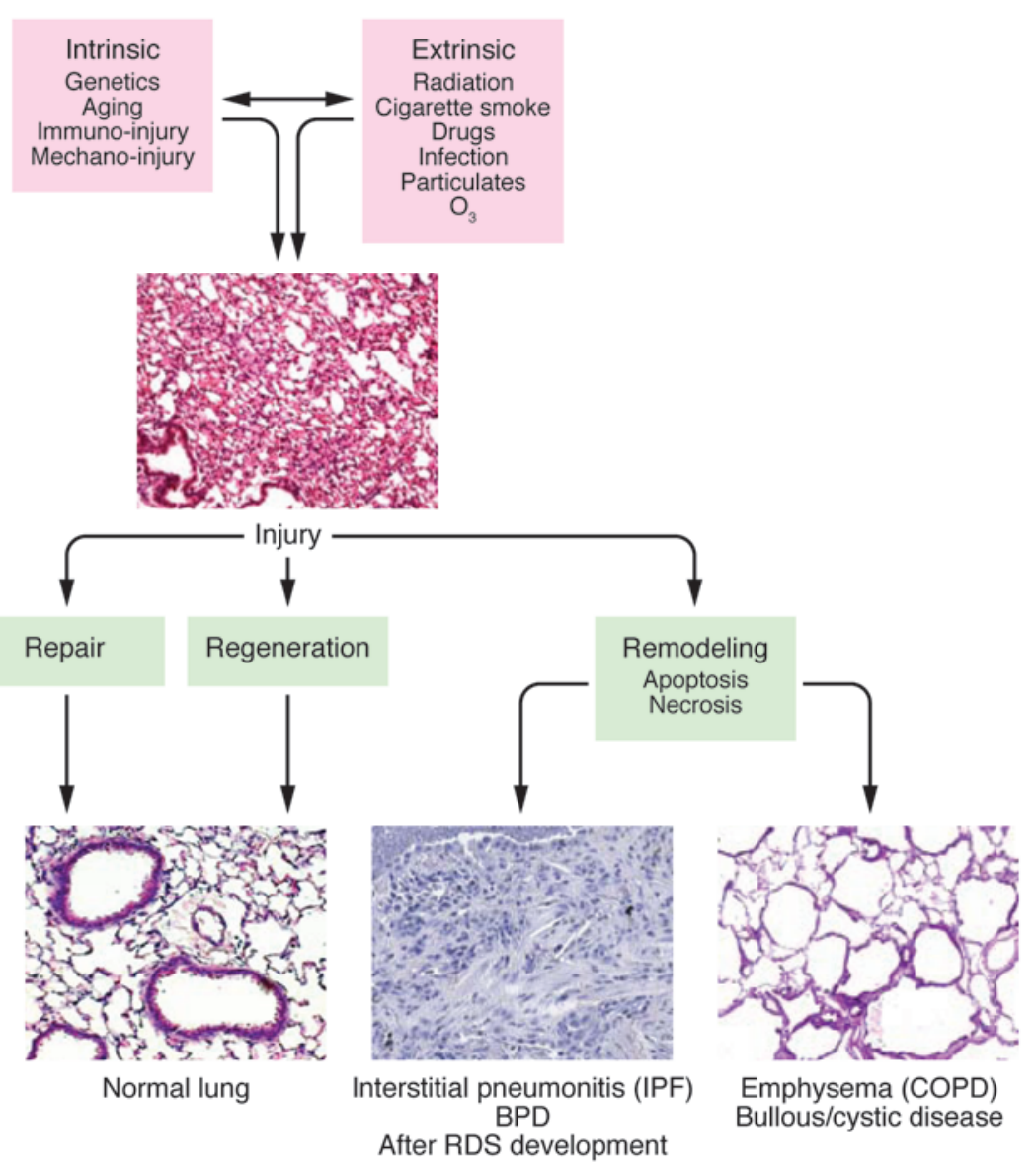

\section{Figure 2}

The 3 R's of lung homeostasis. Schematic model of injury-repair responses in the distal lung. In many cases of parenchymal lung diseases, including pulmonary fibrosis, emphysema, and pulmonary hypertension, both intrinsic (e.g., genetic mutations, mechanical stress, immunological signaling) and extrinsic (e.g., toxins, oxidants) sources of injury combine in a "multiple hit" process to produce resident cell (epithelial or endothelial) dysfunction. Local responses by affected cell populations can preserve normal homeostasis (repair), but, if these are unsuccessful, resident cell death ensues, and a decision either to regenerate (restoring normal architecture and physiology) or to remodel (producing the resultant dysfunctional disease state) is initiated. BPD, bronchopulmonary dysplasia. repair occurs, resulting in the development of a fibrotic scar and/or differentiation to produce an emphysematous pathology. Overall, this scenario suggests a central role for alveolar epithelium in disease pathogenesis and one in which chronic injury and defective repair leads to progressive remodeling reminiscent of abnormal wound repair.

There are several excellent examples demonstrating the critical role of the epithelial cell in initiation of parenchymal lung disease, including monogenetic diseases such Hemansky-Pudlak syndrome and Nieman-Pick disease as well patients with missense or deletion mutations in lung-specific genes such as surfactant protein $\mathrm{C}$ (SFTPC) (40-42) and ABC, subfamily A, member 3 (ABCA3) (43, 44). These diseases are characterized by dysfunctional AEC2 cells and associated with a fibrotic pathology. In mice, targeted ablation of AEC2 cells results in extensive lung fibrosis. (45). Moreover, expression of mutant forms of SFTPC associated with human respiratory phenotypes including fibrosis produces a deleterious cellular injury response, including activation of ER stress, apoptosis, and proinflammatory cytokine expression, which is similar to the phenotype of patients with mutations in SFTPC and SFTPA (46-50). Idiopathic interstitial pneumonias associated with mutations in SFTPC or ABCA3 genes are associated with prolonged ER stress and apoptosis of epithelial cells in vitro (41-44, 46, 47). ER stress is also evident in the biopsy specimens of patients with COPD (51). Finally, epithelial injury is a central finding in the lungs of patients with ALI and ARDS and targeted epithelial injury can produce ARDS in animal models (52).
Extensive epithelial cell damage and eventual loss promotes exposure of basal lamina, exudation of serum proteins, and rearrangement of the underlying connective tissue architecture and extracellular matrix (37). In addition, both the distal and proximal lung epithelia can promote an increased secondary inflammatory response, causing pulmonary recruitment of inflammatory effector cells such as lymphocytes, macrophages, and neutrophils to the interstitium and airways. Commensurate alterations in cytokine and chemokine expression patterns further amplify the injury (53, 54). Although chronic inflammation was traditionally viewed as a key to the pathogenesis of fibrotic and obstructive lung disease, it is not a prominent feature in biopsies of patients with interstitial pulmonary fibrosis/usual interstitial pneumonia (IPF/UIP), and antiinflammatory and immunosuppressive therapies have been largely unsuccessful, leading to reassessment of the role of inflammation in this disorder (37). Similarly, while inflammatory effector cells have clearly been found in the airways of patients with COPD (55), it is unclear whether the observed inflammation represents a primary process or a secondary response to other stimuli.

This has also been shown to occur in lung epithelia in a variety of pathologic conditions. Bronchoalveolar lavage fluid from patients with ARDS can induce apoptosis in airway epithelial cells in culture via activation of the Fas-FasL pathway, and this effect can be inhibited by the use of caspase inhibitors (52). Markers of epithelial cell apoptosis have been demonstrated in patients with idiopathic pulmonary fibrosis $(46,47,56,57)$, and, though diverse, extrinsic signaling pathways also clearly contribute to epithelial 


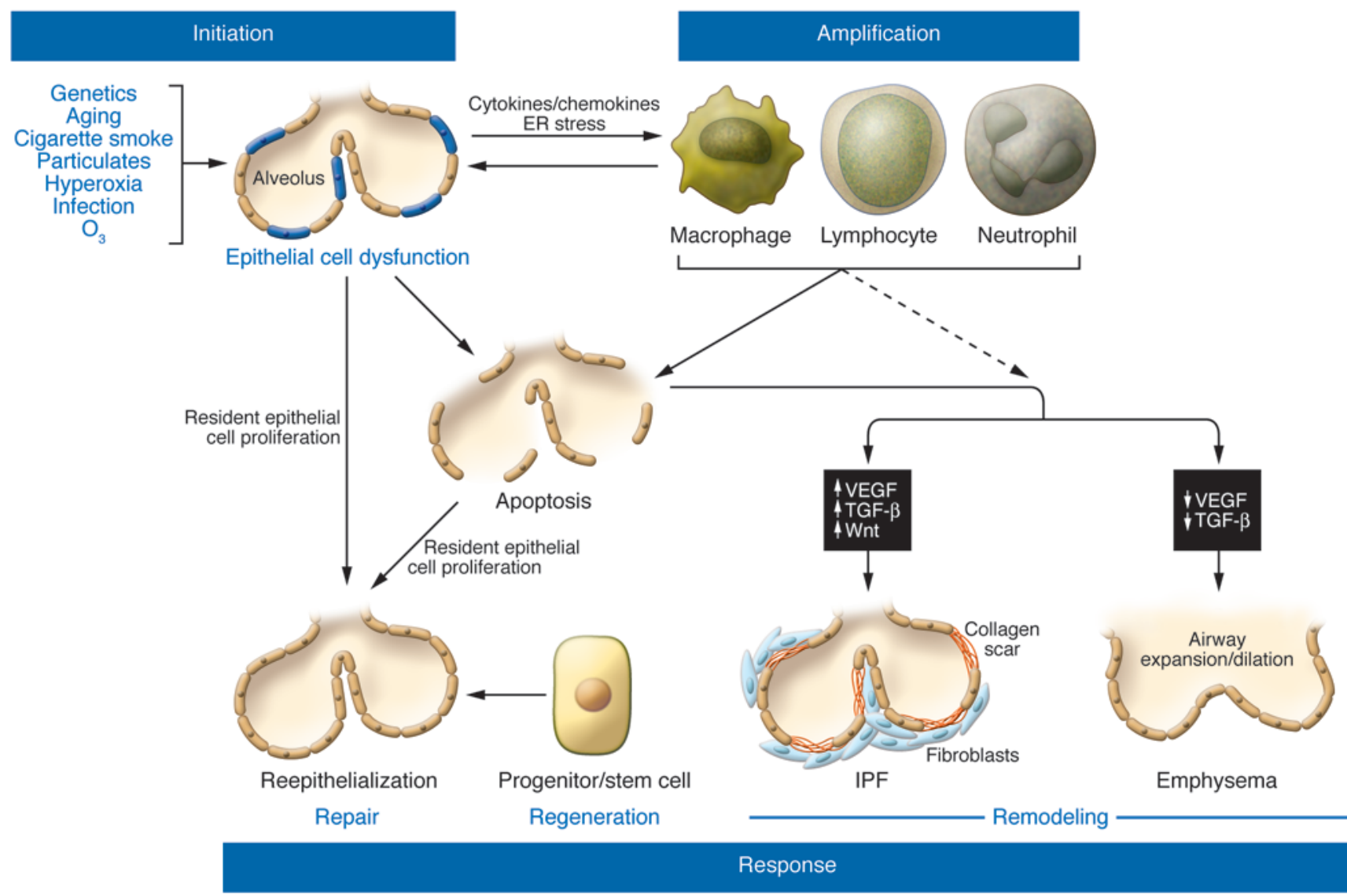

Figure 3

The epithelial-mesenchymal wound model for lung injury and repair. In the initiation stage, multiple microinjuries damage and activate alveolar epithelial cells. In the amplification stage, the resulting ER stress and other activating signals in AEC cells can promote release of chemokines/ cytokines, promoting migration of inflammatory effector cells into the distal lung. Persistent activation and/or ongoing ER stress can result in apoptosis and AEC cell drop-out. In the response stage, in order to restore the denuded epithelial surface, repair via reepithelialization is attempted using either local proliferation/transdifferentiation of AT2 cells or regional expansion of progenitor cell populations such as BASCs (regeneration). In the absence of successful reepithelialization (repair or regeneration), remodeling is initiated. In a fibrotic phenotype (e.g., IPF), the local milieu (including high TGF- $\beta$ and high VEGF levels) promotes proliferation of fibroblasts, activation of myofibroblasts, increases in basement membrane disruption, and neovascularization, with resulting formation of a collagen scar. In emphysema, under different local conditions (including low VEGF and low TGF- $\beta$ levels), airspace enlargement takes place.

cell demise. Markers of apoptosis have also been found in COPD, in which, in contrast to ARDS or pulmonary fibrosis, the loss of epithelial cells is associated with an ongoing destructive remodeling process fueled in part by aberrant immune responses to environmental triggers, such as cigarette smoke, and is dependent on distinct changes in extrinsic growth factor expression and signaling molecules, including VEGF and TGF- $\beta 1(58,59)$. Additional pathways, including those triggered by exogenous stimuli and those intrinsic to epithelial cells such as prolonged activation of the unfolded protein response, can also contribute significantly to improper repair and regeneration of lung epithelium $(35,60-62)$.

Taken together, these data indicate that the integrative response of the adult lung epithelium to apoptotic signals is important in disease initiation. According to the current model of lung injury and regeneration, there is increased sensitivity of epithelial cells to apoptotic injury and a relative resistance of fibroblast and inflammatory cell populations to these same processes, all of which leads to a reduction in epithelial repair and regeneration, which tips the balance toward aberrant remodeling (56). Thus, while promotion of apoptosis is important for culling, shaping, and limiting inflammatory cell populations, inappropriate levels of epithelial apoptosis are inhibitory to regeneration and, if not controlled, lead to a deleterious remodeling process and respiratory insufficiency.

\section{Reparative responses to injury}

Based on these observations, reparative reepithelialization is likely to be crucial to the prevention of pathological lung remodeling. During postnatal life, the ability of the lung epithelium to regenerate itself has been well established, but how this occurs is a subject of much debate. Future regenerative therapies could promote local proliferation of remaining undamaged epithelium, activate a resident progenitor population, use exogenous lung progenitors to repair damaged or diseased lungs, or use a combination of any of these mechanisms.

Respiratory gas exchange is mediated by alveolar epithelium, notably AEC 1 and AEC 2 cells. Within the alveolus, AEC2 cells may 

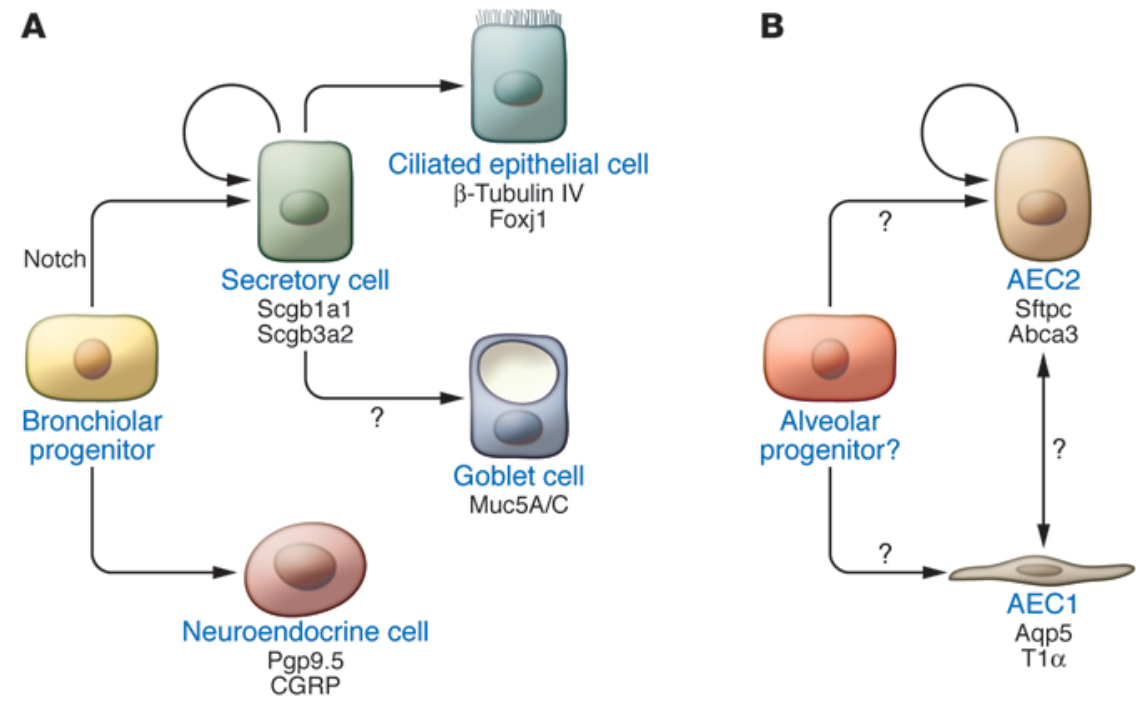

Figure 4

Cell lineages involved in repair and regeneration of the adult lung epithelium. (A) In the adult proximal or bronchiolar epithelium, cells expressing Sox2, p63, and Krt5 are thought to be progenitors capable of regenerating injured or denuded airway epithelium. Whether these markers denote unique, distinct, or overlapping progenitor populations is unknown, but data show that p63 and Krt5 mark a more proximal population of cells than Sox2 alone, which is expressed throughout tracheal and bronchiolar regions $(15,70)$. Notch signaling is known to regulate the decision to generate secretory lineages (i.e., Scgb1a1+) versus neuroendocrine cells within the proximal airways. The secretory lineage has also been shown to generate both ciliated epithelium ( $\beta$-tubulin $\mathrm{IV}^{+}$) as well as goblet cells (Muc5A/C+ ) after injury to the bronchiolar epithelium. (B) In the alveolar epithelium, less is known about the relationship between the two major cell types, AEC1 and AEC2 cells. There is some in vitro evidence that AEC2 cells can act as progenitors to repopulate lost AEC1 and AEC2 cells after injury (63). Additional genetic and molecular data are needed to assess whether there is a resident alveolar progenitor or whether AEC2 cells are facultative progenitors. Aqp5, aquaporin 5; CGRP, Calcitonin gene-related peptide; Pgp9.5, protein gene product 9.5; $\mathrm{T} 1 \alpha$, podoplanin.

act as facultative progenitors, with the capability to both replace themselves as well as differentiate into AEC1 cells after injury (ref. 63 and Figure 4). The importance of AEC2 cells in alveolar repair and regeneration is underscored by animal models, showing that, in bleomycin-induced lung injury, intratracheal delivery of a purified population of syngeneic AEC2 cells was sufficient to block fibrotic lung remodeling (64). However, because AEC2 cells appear to be at the center of the many of the injury pathways, perturbation of these cells also contains the risk of promoting undesirable responses, such as increased fibrosis or increased apoptosis. Epithelial cell loss could also activate a regional population of as yet poorly characterized progenitors within the alveolar or airway epithelium (Figure 4). Although the case for regional progenitor cell activity is fairly robust for the trachea and bronchiolar regions of the lung $(5,7)$, less is known about whether a progenitor population is required in alveolar regeneration, though there are data suggesting that AEC2 cells can act as resident progenitors for alveolar epithelium (63).

The concept that there are different progenitors populations within spatially distinct regions in the adult lung, rather than a "master" lung stem/progenitor cell capable of repairing any lost epithelial cell, has become more accepted from recent fate-mapping approaches $(6,65)$. Although originally an attractive idea, there is little genetic data supporting the existence of a master lung endoderm progenitor in the adult, and the only cell popula- tion that has been experimentally shown to generate both distal/alveolar as well as proximal airway epithelium are embryonic lung endoderm progenitors located in the distal lung endoderm prior to E13.5 (6).

Many lessons related to repair in the distal lung can be learned from the study of airway epithelium. In the bronchiolar region of the adult lung, several recent studies have pointed to a subset of Clara cells that act as resident progenitors, important for repair and regeneration. Clara cells are the most abundant nonciliated secretory cell lineage within the bronchiolar and tracheal airways. Treatment of mice with an intraperitoneal injection of naphthalene induces dramatic and reproducible destruction of the majority of Clara cells due to the expression of the cytochrome P450 isoform Cyp2f2 $(4,66)$. However, a small population of Clara cells, called variant Clara (Clara ${ }^{\text {var }}$ ) cells, is resistant to naphthalene treatment and appears to repopulate the bronchiolar epithelium (Figure 4 and refs. 4, 66, 67). The essential requirement of Clara cells in repopulating the bronchiolar airways was demonstrated by Reynolds et al. who developed a mouse model in which Clara cells could be specifically ablated (66). Loss of these cells prevented repair of the bronchiolar airways after naphthalene-induced airway injury. Dramatic loss of Clara cells in the lung results in activation of Claravar cells, which are located in the bronchioalveolar duct junction (BADJ) and next to neuroendocrine cells $(4,67)$. Clara cells are also capable of generating ciliated epithelium after injury, which further supports their ability to act as progenitors in the bronchiolar epithelium (ref. 65 and Figure 4). Some Clara cells, possibly the Claravar population, appear to express the surfactant protein Sftpc and are expanded in lung injury models, including bleomycin and naphthalene injury (68, 69). The expression of Sftpc by such cells suggests that they may be related to the previously reported bronchioalveolar stem cell (BASC) located in the BADJ region of the lung, which also expresses both Sftpc and Scgb1a1 (also known as Clara cell 10-kilodalton protein [CC10]) (69). Studies have shown that BASCs can generate both bronchiolar as well as alveolar lineages ex vivo (69). However, recent genetic fate-mapping analysis shows that Scgb1a-expressing Clara cells are unable to generate alveolar epithelium either during homeostasis or after airway injury (65). The discrepancy in these findings could be due to the adaptation of isolated lung epithelium in in vitro culture or to other technical limitations, including the type of injury used in these studies $(65,69)$.

Although not definitive, the increased numbers of BASCs in naphthalene-treated mutant K-ras-expressing mice suggests that BASCs may contribute to lung tumor formation (69). Despite this intriguing concept, recent data has conflicted with the presence of a true Scgb $11^{+} / \mathrm{Sftpc}^{+}$double-positive BASC population capable of generating both alveolar and bronchiolar epithelium. Using an inducible Scgb1a1-creERT2 mouse knock-in line, Raw- 
lins et al. has shown that Scgb1a1-expressing cells do not generate alveolar epithelium during lung homeostasis or after injury (65). Whether Sftpc cells can generate bronchiolar epithelium or other alveolar epithelial cells, including AEC1, has not yet been determined using genetic fate-mapping approaches. Thus, current data does implicate the presence of spatially restricted progenitors within the adult lung, but as yet the presence of a master lung stem cell is still debatable.

As outlined in previous sections, lung epithelial cell types are patterned in a distinct proximal-distal manner required for their specific functions. For example, AEC2 cells are localized to distal alveolar regions and specialize in surfactant production, while microparticle clearance is performed by ciliated epithelium in more proximal airways. Epithelia in the bronchial and tracheal airways consist of several cell types, including nonciliated/secretary Clara cells, ciliated epithelia, mucous cells, basal cells, and neuroendocrine cells (Figure 4). Besides Clara ${ }^{\text {var }}$ cells, basal cells have also been implicated as progenitors in the tracheal and proximal airways. Basal cells underlie the entire tracheal airway of the rodent and human lung and are characterized by their expression of transformation-related protein 63 (Tp63, also known as p63) and the genes keratin 14 (Krt14) and $K r t 5$ (7). Using Krt5-creERT2 and Krt14creERT2 fate-mapping mouse lines, $\mathrm{Krt}^{+}$and $\mathrm{Krt} 14^{+}$airway progenitors have been shown to generate Clara and ciliated epithelium during tracheal homeostasis $(5,7)$. Sox 2 also marks progenitors within the upper airways of rodents and humans, but it remains unclear whether Sox $2^{+}$progenitors are the same, distinct from, or overlapping with the $\mathrm{Tp} 63^{+}, \mathrm{Krt5}^{+}$, or $\mathrm{Krt} 14^{+}$progenitor populations (Table 1 and refs. 14, 15, 70). Future studies using additional tools, including conditional cre lines driven from the Sox2, Tp63, and other important marker loci, should help to clarify the hierarchy of upper airway progenitors in the mouse and human lung.

Although many studies have provided evidence of resident progenitors within the adult lung capable of responding to injury, some models of lung injury and postnatal growth have shown that simple cell proliferation can repopulate lost cells within the adult lung. The pneumonectomy model of lung reduction and regrowth in the mouse produces an extensive activation of cell proliferation that leads to restoration of pulmonary function, primarily through global cell proliferative responses and proportional regrowth of the remaining lobes of the lung (71-77). This process is likely similar to what occurs in the liver, in which extensive cellular loss results in regrowth almost solely due to proliferation of remaining cell populations, including hepatocytes (78). This model does not exclude the presence and activity of a progenitor population in either organ; indeed, a previous report identified potential progenitor populations in the liver capable of limited regenerative ability (79). Interestingly, the molecular pathways involved in liver regrowth are the same as those involved in progenitor cell activation in the lung, including the Wnt/ $\beta$-catenin, Fgf, and Bmp signaling pathways (78). Thus, the molecular response to lung injury and regeneration likely involves the same core set of pathways and factors irrespective of whether it involves tissue-specific progenitors or cell replacement through proliferation and redifferentiation of undamaged cells.

Whether lung regeneration involves activation of spatially distinct progenitors, repopulation of damaged epithelia through cell proliferation, or both, the molecular pathways that regulate this regenerative response in the adult lung are not well understood. However, analysis of developmentally important pathways such as Wnt/ $\beta$-catenin signaling in the adult lung has revealed interesting and at times opposing effects on lung repair and regeneration. Previous studies have shown that $\mathrm{Wnt} / \beta$-catenin signaling activity is activated in the adult lung through loss of the transcription factor Gata6 (68). Moreover, forced activation of Wnt/ $\beta$-catenin signaling using a stabilized form of $\beta$-catenin leads to a significant expansion of the Claravar progenitor population $(68,80)$. Despite these findings, which suggest a critical role for $\mathrm{Wnt} / \beta$-catenin in promoting bronchiolar progenitor expansion, $\beta$-catenin is not required for proper Clara cell regeneration in the adult mouse lung (81). During development, Wnt/ $\beta$-catenin signaling activity is highest in the distal airway epithelium, which will give rise to alveolar epithelium postnatally, suggesting that its role in promoting epithelial regeneration may be more potent in this cell lineage. Such a finding could have important clinical implications for lung repair, since activators of Wnt signaling including lithium salts are already FDA-approved drugs. However, in contrast to the potent role of Wnt signaling in promoting lung bronchiolar progenitor expansion, recent studies using an inhibitor of Wnt/CBP interactions show that blocking some aspects of Wnt signaling actually reduces the fibrotic response after bleomycin-induced lung injury (82). This is supported by finding that Wnt signaling is increased in myofibroblasts of IPF patients $(83,84)$. Thus, a note of caution is warranted in using either activators or inhibitors of these pathways as their effects are very context dependent, and chronic activation could lead to a decrease in repair potential by promoting a fibrotic response.

\section{Summary and conclusions}

The ultimate goal of any strategy of lung repair is to repopulate lung airways with the appropriate epithelial- and mesenchymal-derived cells required to form functional bronchi and alveolar units. Many of the pathways discussed here could be harnessed therapeutically to promote endogenous repair and regeneration. However, the lung offers unique challenges for applying systemic therapy, given both the level of complexity and the intricate tissue architecture. As for the application of tissue engineering to lung regenerative therapies, the sheer number of different cell types within the mature lung as well as the intimate interactions between them suggests that novel technologies will have to be developed to begin to generate lung tissue that is correctly patterned ex vivo. Encouraging and significant advances have been made in rodent models using decellularized lung scaffolds (85). In addition, recent findings in the field of iPS cells as well as direct reprogramming of differentiated cells using transcription factors suggest that our ability to control cell lineage differentiation and fate is increasing (86-88). Such technical leaps may make it possible some day to generate lung epithelium from patient-specific iPS cells or, through as yet unknown methods, to directly reprogram patient cells into lung epithelium, bypassing the requirement for iPS cell generation. These new techniques will have a profound impact on manipulating endogenous progenitors to promote repair and regeneration. Just as the identification of the factors that generate iPS cells from fibroblasts came from basic developmental biology studies, further understanding of the mechanisms underlying lung epithelial development and differentiation are critical for promoting endogenous lung regeneration as well as lung epithelial lineage differentiation in pluripotent stem cells. A combination of increased basic understanding of the molecular pathways that promote endogenous repair and regeneration as well as advances in bioengineered materials should lead 
to further insights into how such cells can be harnessed for lung repair and regeneration.

\section{Acknowledgments}

We apologize for any omissions of references due to space limitations. Studies in the Morrisey and Beers laboratories are supported by funding from the NIH (HL071589, HL087825, and HL100405 to E.E. Morrisey and P30 ES015857, HL19737, and HL087177 to M.F. Beers).
Address correspondence to: Edward E. Morrisey, University of Pennsylvania, 956 BRB II/III, 421 Curie Blvd., Philadelphia, Pennsylvania 19104, USA. Phone: 215.573.3010; Fax: 215.573.2094; E-mail: emorrise@mail.med.upenn.edu. Or to: Michael F. Beers, University of Pennsylvania, Pulmonary and Critical Care Division, Vernon and Shirley Hill Pavilion, 380 S. University Avenue, Room H410F, Philadelphia, Pennsylvania 19104-4539, USA. Phone: 215.898.9106; Fax: 215.746.5985; E-mail: mfbeers@mail.med.upenn.edu.
1. Cardoso WV, Lu J. Regulation of early lung morphogenesis: questions, facts and controversies. Development. 2006;133(9):1611-1624.

2. Cardoso WV, Whitsett JA. Resident cellular components of the lung: developmental aspects. Proc Am Thorac Soc. 2008;5(7):767-771.

3. Morrisey EE, Hogan BL. Preparing for the first breath: genetic and cellular mechanisms in lung development. Dev Cell. 2010;18(1):8-23.

4. Giangreco A, Reynolds SD, Stripp BR. Terminal bronchioles harbor a unique airway stem cell population that localizes to the bronchoalveolar duct junction. Am J Pathol. 2002;161(1):173-182.

5. Hong KU, Reynolds SD, Watkins S, Fuchs E, Stripp BR. Basal cells are a multipotent progenitor capable of renewing the bronchial epithelium. $\mathrm{Am}$ J Pathol. 2004;164(2):577-588.

6. Rawlins EL, Clark CP, Xue Y, Hogan BL. The Id2+ distal tip lung epithelium contains individual multipotent embryonic progenitor cells. Development. 2009; 136(22):3741-3745.

7. Rock JR, et al. Basal cells as stem cells of the mouse trachea and human airway epithelium. Proc Natl Acad Sci US A. 2009;106(31):12771-12775.

8. Warburton D, Perin L, Defilippo R, Bellusci S, Shi W, Driscoll B. Stem/progenitor cells in lung development, injury repair, and regeneration. Proc Am Thorac Soc. 2008;5(6):703-706.

9. Crosby LM, Waters CM. Epithelial repair mechanisms in the lung. Am J Physiol Lung Cell Mol Physiol. 2010;298(6):L715-L731.

10. Shannon JM, Hyatt BA. Epithelial-mesenchymal interactions in the developing lung. Annu Rev Physiol. 2004;66:625-645.

11. Shannon JM, Gebb SA, Nielsen LD. Induction of alveolar type II cell differentiation in embryonic tracheal epithelium in mesenchyme-free culture. Development. 1999;126(8):1675-1688.

12. Lu Y, Thomson JM, Wong HY, Hammond SM, Hogan BL. Transgenic over-expression of the microRNA miR-17-92 cluster promotes proliferation and inhibits differentiation of lung epithelial progenitor cells. Dev Biol. 2007;310(2):442-453.

13. Okubo T, Knoepfler PS, Eisenman RN, Hogan BL. Nmyc plays an essential role during lung development as a dosage-sensitive regulator of progenitor cell proliferation and differentiation. Development. 2005; 132(6):1363-1374

14. Que J, Luo X, Schwartz RJ, Hogan BL. Multiple roles for Sox2 in the developing and adult mouse trachea. Development. 2009;136(11):1899-1907.

15. Que J, et al. Multiple dose-dependent roles for Sox 2 in the patterning and differentiation of anterior foregut endoderm. Development. 2007;134(13):2521-2531.

16. Moon RT. Wnt/beta-catenin pathway. Sci STKE. 2005;2005(271): $\mathrm{cm} 1$.

17. Shu W, et al. Wnt/beta-catenin signaling acts upstream of N-myc, BMP4, and FGF signaling to regulate proximal-distal patterning in the lung. Dev Biol. 2005;283(1):226-239.

18. Okubo T, Hogan BL. Hyperactive Wnt signaling changes the developmental potential of embryonic lung endoderm. J Biol. 2004;3(3):11.

19. Cohen ED, Ihida-Stansbury K, Lu MM, Panettieri RA, Jones PL, Morrisey EE. Wnt signaling regulates smooth muscle precursor development in the mouse lung via a tenascin C/PDGFR pathway. J Clin
Invest. 2009;119(9):2538-2549.

20. Goss AM, et al. Wnt $2 / 2 \mathrm{~b}$ and beta-catenin signaling are necessary and sufficient to specify lung progenitors in the foregut. Dev Cell. 2009;17(2):290-298.

21. Harris-Johnson KS, Domyan ET, Vezina CM, Sun $\mathrm{X}$. beta-Catenin promotes respiratory progenitor identity in mouse foregut. Proc Natl Acad Sci US A. 2009;106(38):16287-16292.

22. Mucenski ML, et al. beta-Catenin is required for specification of proximal/distal cell fate during lung morphogenesis. J Biol Chem. 2003;278(41):40231-40238.

23. Cohen ED, et al. Wnt/beta-catenin signaling promotes expansion of Isl-1-positive cardiac progenitor cells through regulation of FGF signaling. J Clin Invest. 2007;117(7):1794-1804.

24. Sekine K, et al. Fgf10 is essential for limb and lung formation. Nat Genet. 1999;21(1):138-141.

25. Min H, et al. Fgf-10 is required for both limb and lung development and exhibits striking functional similarity to Drosophila branchless. Genes Dev. 1998; 12(20):3156-3161.

26. Arman E, Haffner-Krausz R, Gorivodsky M, Lonai P. Fgfr 2 is required for limb outgrowth and lung-branching morphogenesis. Proc Natl Acad Sci U S A. 1999; 96(21):11895-11899.

27. White AC, Xu J, Yin Y, Smith C, Schmid G, Ornitz DM. FGF9 and SHH signaling coordinate lung growth and development through regulation of distinct mesenchymal domains. Development. 2006; 133(8):1507-1517.

28. Colvin JS, White AC, Pratt SJ, Ornitz DM. Lung hypoplasia and neonatal death in Fgf9-null mice identify this gene as an essential regulator of lung mesenchyme. Development. 2001;128(11):2095-2106.

29. Lu MM, Yang H, Zhang L, Shu W, Blair DG, Morrisey EE. The bone morphogenic protein antagonist gremlin regulates proximal-distal patterning of the lung. Dev Dyn. 2001;222(4):667-680.

30. Weaver M, Yingling JM, Dunn NR, Bellusci S, Hogan BL. Bmp signaling regulates proximal-distal differentiation of endoderm in mouse lung development. Development. 1999;126(18):4005-4015.

31. Eblaghie MC, Reedy M, Oliver T, Mishina Y, Hogan $B L$. Evidence that autocrine signaling through Bmpr1a regulates the proliferation, survival and morphogenetic behavior of distal lung epithelial cells. Dev Biol. 2006;291(1):67-82.

32. Shu W, Lu MM, Zhang Y, Tucker PW, Zhou D, Morrisey EE. Foxp2 and Foxp1 cooperatively regulate lung and esophagus development. Development.2007; 134(10):1991-2000.

33. Wert SE, Dey CR, Blair PA, Kimura S, Whitsett JA. Increased expression of thyroid transcription factor-1 (TTF-1) in respiratory epithelial cells inhibits alveolarization and causes pulmonary inflammation. Dev Biol. 2002;242(2):75-87.

34. Liu C, Ikegami M, Stahlman MT, Dey CR, WhitsettJA. Inhibition of alveolarization and altered pulmonary mechanics in mice expressing GATA-6. Am J Physiol Lung Cell Mol Physiol. 2003;285(6):L1246-L1254.

35. Selman M, Pardo A, Kaminski N. Idiopathic pulmonary fibrosis: aberrant recapitulation of developmental programs? PLoS Med. 2008;5(3):e62.

36. Ayo DS, Aughenbaugh GL, Yi ES, Hand JL, Ryu JH. Cystic lung disease in Birt-Hogg-Dube syndrome. Chest. 2007;132(2):679-684.

37. Thannickal VJ, Toews GB, White ES, Lynch JP 3rd,
Martinez FJ. Mechanisms of pulmonary fibrosis. Annu Rev Med. 2004;55:395-417.

38. Selman M, Pardo A. Role of epithelial cells in idiopathic pulmonary fibrosis: from innocent targets to serial killers. Proc Am Thorac Soc. 2006;3(4):364-372.

39. Budhiraja R, Tuder RM, Hassoun PM. Endothelial dysfunction in pulmonary hypertension. Circulation. 2004;109(2):159-165.

40. Nogee LM, Dunbar AE 3rd, Wert S, Askin F, Hamvas A, Whitsett JA. Mutations in the surfactant protein $\mathrm{C}$ gene associated with interstitial lung disease. Chest. 2002;121(3 suppl):20S-21S.

41. Nogee LM, Dunbar AE 3rd, Wert SE, Askin F, Hamvas A, Whitsett JA. A mutation in the surfactant protein $\mathrm{C}$ gene associated with familial interstitial lung disease. N Engl J Med. 2001;344(8):573-579.

42. Thomas AQ, et al. Heterozygosity for a surfactant protein $\mathrm{C}$ gene mutation associated with usual interstitial pneumonitis and cellular nonspecific interstitial pneumonitis in one kindred. Am J Respir Crit Care Med. 2002;165(9):1322-1328.

43. Bullard JE, Wert SE, Whitsett JA, Dean M, Nogee LM. ABCA3 mutations associated with pediatric interstitial lung disease. Am J Respir Crit Care Med. 2005;172(8):1026-1031.

44. Shulenin S, Nogee LM, Annilo T, Wert SE, Whitsett JA, Dean M. ABCA3 gene mutations in newborns with fatal surfactant deficiency. NEngl J Med. 2004; 350(13):1296-1303.

45. Sisson TH, et al. Targeted injury of type II alveolar epithelial cells induces pulmonary fibrosis. Am J Respir Crit Care Med. 2010;181(3):254-263.

46. Korfei M, et al. Epithelial endoplasmic reticulum stress and apoptosis in sporadic idiopathic pulmonary fibrosis. Am J Respir Crit Care Med. 2008; 178(8):838-846.

47. Lawson WE, et al. Endoplasmic reticulum stress in alveolar epithelial cells is prominent in IPF: association with altered surfactant protein processing and herpesvirus infection. Am J Physiol Lung Cell Mol Physiol. 2008;294(6):L1119-L1126.

48. Maitra M, Wang Y, Gerard RD, Mendelson CR, Garcia CK. Surfactant protein A2 mutations associated with pulmonary fibrosis lead to protein instability and endoplasmic reticulum stress. J Biol Chem. 2010;285(29):22103-22113.

49. Mulugeta S, Maguire JA, Newitt JL, Russo SJ, Kotorashvili A, Beers MF. Misfolded BRICHOS SP-C mutant proteins induce apoptosis via caspase-4and cytochrome c-related mechanisms. Am J Physiol Lung Cell Mol Physiol. 2007;293(3):L720-L729.

50. Mulugeta S, Nguyen V, Russo SJ, Muniswamy M, Beers MF. A surfactant protein C precursor protein BRICHOS domain mutation causes endoplasmic reticulum stress, proteasome dysfunction, and caspase 3 activation. Am J Respir Cell Mol Biol. 2005; 32(6):521-530

51. Malhotra D, et al. Heightened endoplasmic reticulum stress in the lungs of patients with chronic obstructive pulmonary disease: the role of $\mathrm{Nrf2}$ regulated proteasomal activity. Am J Respir Crit Care Med. 2009;180(12):1196-1207.

52. Matute-Bello G, et al. Soluble Fas ligand induces epithelial cell apoptosis in humans with acute lung injury (ARDS). J Immunol. 1999;163(4):2217-2225.

53. Strieter RM, Gomperts BN, Keane MP. The role of CXC chemokines in pulmonary fibrosis. J Clin 
Invest. 2007;117(3):549-556.

54. Barnes PJ. The cytokine network in asthma and chronic obstructive pulmonary disease. J Clin Invest. 2008;118(11):3546-3556.

55. Hogg JC, et al. The nature of small-airway obstruction in chronic obstructive pulmonary disease. NEngl J Med. 2004;350(26):2645-2653.

56. Uhal BD, Joshi I, Hughes WF, Ramos C, Pardo A, Selman M. Alveolar epithelial cell death adjacent to underlying myofibroblasts in advanced fibrotic human lung. Am J Physiol. 1998;275(6 pt 1):L1192-L1199.

57. Barbas-Filho JV, Ferreira MA, Sesso A, Kairalla RA, Carvalho CR, Capelozzi VL. Evidence of type II pneumocyte apoptosis in the pathogenesis of idiopathic pulmonary fibrosis (IFP)/usual interstitial pneumonia (UIP). J Clin Pathol. 2001;54(2):132-138.

58. Kasahara Y, et al. Inhibition of VEGF receptors causes lung cell apoptosis and emphysema. J Clin Invest. 2000;106(11):1311-1319.

59. Tuder RM, Kasahara Y, Voelkel NF. Inhibition of vascular endothelial growth factor receptors causes emphysema in rats. Chest. 2000;117(5 suppl 1):281S.

60. Green DR, Kroemer G. The pathophysiology of mitochondrial cell death. Science. 2004; 305(5684):626-629.

61. Tait SW, Green DR. Mitochondria and cell death: outer membrane permeabilization and beyond. Nat Rev Mol Cell Biol. 2010;11(9):621-632.

62. Xu C, Bailly-Maitre B, Reed JC. Endoplasmic reticulum stress: cell life and death decisions. J Clin Invest. 2005;115(10):2656-2664.

63. Adamson IY, Bowden DH. Derivation of type 1 epithelium from type 2 cells in the developing rat lung. Lab Invest. 1975;32(6):736-745.

64. Serrano-Mollar A, Nacher M, Gay-Jordi G, Closa D, Xaubet A, Bulbena O. Intratracheal transplantation of alveolar type II cells reverses bleomycin-induced lung fibrosis. Am J Respir Crit Care Med. 2007; 176(12):1261-1268.

65. Rawlins EL, et al. The role of Scgb1a1+ Clara cells in the long-term maintenance and repair of lung airway, but not alveolar, epithelium. Cell Stem Cell.
2009;4(6):525-534.

66 . Reynolds SD, et al. Conditional clara cell ablation reveals a self-renewing progenitor function of pulmonary neuroendocrine cells. Am J Physiol Lung Cell Mol Physiol. 2000;278(6):L1256-L1263.

67. Hong KU, Reynolds SD, Giangreco A, Hurley CM, Stripp BR. Clara cell secretory protein-expressing cells of the airway neuroepithelial body microenvironment include a label-retaining subset and are critical for epithelial renewal after progenitor cell depletion. Am J Respir Cell Mol Biol. 2001;24(6):671-681.

68. Zhang Y, et al. A Gata6-Wnt pathway required for epithelial stem cell development and airway regeneration. Nat Genet. 2008;40(7):862-870.

69. Kim CF, et al. Identification of bronchioalveolar stem cells in normal lung and lung cancer. Cell. 2005; 121(6):823-835.

70. Tompkins DH, et al. Sox 2 is required for maintenance and differentiation of bronchiolar Clara, ciliated, and goblet cells. PLoS One. 2009;4(12):e8248.

71. Voswinckel R, et al. Characterisation of post-pneumonectomy lung growth in adult mice. Eur Respir J. 2004;24(4):524-532.

72. Hoffman AM, et al. Matrix modulation of compensatory lung regrowth and progenitor cell proliferation in mice. Am J Physiol Lung Cell Mol Physiol. 2010;298(2):L158-L168.

73. Nolen-Walston RD, et al. Cellular kinetics and modeling of bronchioalveolar stem cell response during lung regeneration. Am J Physiol Lung Cell Mol Physiol. 2008;294(6):L1158-L1165.

74. Leuwerke SM, Kaza AK, Tribble CG, Kron IL, Laubach VE. Inhibition of compensatory lung growth in endothelial nitric oxide synthase-deficient mice. Am J Physiol Lung Cell Mol Physiol. 2002; 282(6):L1272-L1278.

75. Kaza AK, et al. Epidermal growth factor receptor up-regulation is associated with lung growth after lobectomy. Ann Thorac Surg. 2001;72(2):380-385.

76. Kaza AK, et al. Retinoic acid enhances lung growth after pneumonectomy. Ann Thorac Surg. 2001; 71(5):1645-1650.
77. Kaza AK, et al. Epidermal growth factor augments postpneumonectomy lung growth.J Thorac Cardiovasc Surg. 2000;120(5):916-921.

78. Michalopoulos GK. Liver regeneration.J Cell Physiol. 2007;213(2):286-300

79. Fausto N, Campbell JS. The role of hepatocytes and oval cells in liver regeneration and repopulation. Mech Dev. 2003;120(1):117-130.

80. Reynolds SD, et al. Conditional stabilization of beta-catenin expands the pool of lung stem cells. Stem Cells. 2008;26(5):1337-1346.

81. Zemke AC, et al. beta-Catenin is not necessary for maintenance or repair of the bronchiolar epithelium. Am J Respir Cell Mol Biol. 2009;41(5):535-543.

82. Henderson WR Jr, et al. Inhibition of Wnt/betacatenin/CREB binding protein (CBP) signaling reverses pulmonary fibrosis. Proc Natl Acad Sci US A. 2010; 107(32):14309-14314

83. Chilosi M, et al. Aberrant Wnt/beta-catenin pathway activation in idiopathic pulmonary fibrosis. Am J Pathol. 2003;162(5):1495-1502.

84. Konigshoff M, et al. Functional Wnt signaling is increased in idiopathic pulmonary fibrosis. PLOS One. 2008;3(5):e2142.

85. Petersen TH, et al. Tissue-engineered lungs for in vivo implantation. Science. 2010;329(5991):538-541.

86. Takahashi $\mathrm{K}$, et al. Induction of pluripotent stem cells from adult human fibroblasts by defined factors. Cell. 2007;131(5):861-872.

87. Meissner A, Wernig M, Jaenisch R. Direct reprogramming of genetically unmodified fibroblasts into pluripotent stem cells. Nat Biotechnol. 2007; 25(10):1177-1181.

88. Takahashi K, Yamanaka S. Induction of pluripotent stem cells from mouse embryonic and adult fibroblast cultures by defined factors. Cell. 2006; 126(4):663-676.

89. Hong KU, Reynolds SD, Watkins S, Fuchs E, Stripp BR. In vivo differentiation potential of tracheal basal cells: evidence for multipotent and unipotent subpopulations. Am J Physiol Lung Cell Mol Physiol. 2004; 286(4):L643-L649. 